Pacific

Journal of

Mathematics

\title{
DILATATION OF MAPS BETWEEN SPHERES
}

Peng Chiakuei and Tang Zizhou 


\title{
DILATATION OF MAPS BETWEEN SPHERES
}

\author{
Peng Chiakuei And Tang Zizhou \\ Dedicated to Professor Wu W.T. on his 80th birthday
}

\begin{abstract}
For a smooth map between spheres, we are concerned with the relation between its homotopy class (topological complexity) and its dilatation (geometrical complexity). This paper (1) generalizes the results of Olivier and Roitberg on the dilatation of Hopf fibrations and the elements of the stable homotopy groups of spheres. (2) Disproves two conjectures of Olivier and Roitberg by showing that $\delta(2,4)<3$ and $\delta(3,4)=2$.
\end{abstract}

\section{Introduction.}

Let $f: S^{m} \rightarrow S^{n}$ be a $C^{1}$ map of the standard unit spheres and let $d f$ be the differential of $f$. Following Olivier [O1], we define $\delta(f)$, the dilatation (or "stretching") of $f$, by the formula

$$
\delta(f)=\sup \{|d f(V)||| V \mid=1\} ;
$$

where $V$ ranges over all unit tangent vectors of $S^{m}$, and $|\cdot|$ is the Euclidean length. Moreover, for a homotopy class $\alpha \in \pi_{m}\left(S^{n}\right)$, we define

$$
\delta(\alpha)=\inf \{\delta(f) \mid[f]=\alpha\},
$$

the infimum taken over all differentiable representatives $f$ of $\alpha$.

As stated in [Gr1], we ask how to estimate a measure of the topological complexity of a map $f: S^{m} \rightarrow S^{n}$ by its geometry. It is natural to measure geometrical complexity of $f$ by its dilatation. The topological complexity of $f$ may be measured by its homotopy class or the Brouwer degree (when the degree makes sense). We should point out in fact that our present paper arose out of continuous attempt to represent homotopy class of spheres by harmonic maps or algebraic maps (cf. [PT2], [PT3] and [PT4]).

Olivier remarked in [O1, p. 387] that the Hopf fibrations $S^{15} \rightarrow S^{8}, S^{7} \rightarrow$ $S^{4}, S^{3} \rightarrow S^{2}$ have dilatation exactly 2 . Furthermore, Roitberg investigated the elements of the stable homotopy groups of spheres $\pi_{k}^{s}=\lim _{n \rightarrow \infty} \pi_{n+k}\left(S^{n}\right)$, and was able to completely analyze the situation for those elements in $\pi_{k}^{s}$ which lie in the image of the stable $J$-homomorphism $\pi_{k}(O) \rightarrow \pi_{k}^{s}$. The dilatation of any such nonzero element was shown to be always 2 [Ro, p. 202]. 
To generalize these results, we try to get information on polynomial maps between spheres. Using Bernstein-Szego Theorem, we can establish in Section 2 a succinct but nice estimate which implies immediately the statements mentioned above.

Theorem 1.1. Let $f: S^{m} \rightarrow S^{n}$ be a polynomial map of algebraic degree $k$. Then $\delta(f) \leq k$.

Several interesting consequences of this theorem will be given in Section 2 .

In Section 3, we make a deep study of problem of obtaining upper bounds for the dilatation invariants of maps $S^{2} \rightarrow S^{2}, S^{3} \rightarrow S^{3}$ and $S^{3} \rightarrow S^{2}$, respectively. In [Ol, p. 389], Olivier used the following definition $(n \geq$ $0, k \geq 0)$ :

$$
\delta(n, k)=\inf \left\{\delta(f) \mid f: S^{n} \rightarrow S^{n} \text { differentiable, } \operatorname{deg}(f)=k\right\}
$$

where $\operatorname{deg}(f)$ denotes the Brouwer degree of the map $f$, which determines completely the homotopy class $[f] \in \pi_{n} S^{n}=Z$. It is worth summarizing the following fundamental characterizations.

(i) $\delta(1, k)=k$ and $\delta(n, k) \leq k[\mathbf{O l}]$;

(ii) $\delta(n, k) \geq \sqrt[n]{k}[\mathbf{O l}]$ and $[\mathbf{G r 2}]$;

(iii) $\delta(n, 2)=2[\mathrm{Ol}]$ and $[\mathbf{H e}]$;

(iv) $\delta(n, k) \geq 2$ for $|k| \geq 2[\mathrm{Ol}]$ and [Gr1].

It was conjectured in $[\mathrm{Ol}]$ that $\delta(2, k)=k$ for $k \geq 0$. Unfortunately, this conjecture will be shown to be false for $k=4$ by the following inequality.

Proposition 1.2. $\delta(2,4) \leq 2 \sqrt{2}$.

It would be interesting to know the exact value of $\delta(2,4)$.

In order to estimate the dilatation of elements in $\pi_{3} S^{3}$, Olivier [O1, p. 389] constructed explicitly a map $f: S^{3} \rightarrow S^{3}$ and stated that this map has dilatation exactly 2 . However, we will show that his map has dilatation more than $\sqrt{\frac{24}{5}}$. In fact, we can prove that any differentiable join: $S^{3}=$ $S^{1} * S^{1} \rightarrow S^{3}$ has dilatation more than 2 . On the other hand, we will construct a family of differentiable maps $S^{3} \rightarrow S^{3}$ of the Brouwer degree 4 whose dilatation approaches 2 . These will imply the following:

Proposition 1.3. $\delta(3,4)=2$.

It was conjectured in [Ro, p. 202] that $\delta(\sigma \circ \tau)=\delta(\sigma) \cdot \delta(\tau)=2 \delta(\tau)$, at least if $\sigma$ is one of the Hopf classes. The final section will provide a counterexample.

Theorem 1.4. Let $\tau \in \pi_{3} S^{3}$ be the homotopy class of the Brouwer degree 2 , and $\sigma \in \pi_{3} S^{2}$ the Hopf class, then $\delta(\sigma \circ \tau)<\sqrt{15}<4=2 \delta(\tau)$. 


\section{Polynomial maps.}

Recall that a map $f: S^{m} \rightarrow S^{n}$ is said to be a polynomial map if it is the restriction to $S^{m}$ of a polynomial map from $R^{m+1}$ to $R^{n+1}$. If, in addition, the polynomials are all homogeneous of degree $k$, then $f$ is called a $k$-form (see, for example, [Wo]). In order to prove Theorem 1.1, we need to use the Bernstein-Szego Inequality which bounds the derivative of a trigonometric polynomial in terms of its supremum norm.

Bernstein-Szego Inequality ([BE, p. 232]). Let

$$
f(\theta)=a_{0}+\sum_{\lambda=1}^{k}\left(a_{\lambda} \cos \lambda \theta+b_{\lambda} \sin \lambda \theta\right), \quad \theta \in[0,2 \pi] .
$$

Then

$$
\left|f^{\prime}(\theta)\right| \leq k \cdot \max _{\theta \in[0,2 \pi]}|f(\theta)| .
$$

We are now in a position to prove Theorem 1.1.

Proof of Theorem 1.1. Suppose that $f: S^{m} \rightarrow S^{n}$ is a polynomial map of degree $k$. Given any point $x$ in $S^{m}$ and a unit tangent vector $V$ to $x$, denote by $S^{1}$ the great circle spanned by $x$ and $V$. By a suitable orthogonal transformation of $R^{m+1}$, we may assume that $x=(1,0, \ldots, 0), V=(0,1, \ldots, 0)$ and

$$
S^{1}=\{(\cos \theta, \sin \theta, 0, \ldots, 0) \mid 0 \leq \theta \leq 2 \pi\} .
$$

Observe that any orthogonal transformation preserves the degree of the polynomials.

Furthermore, by a suitable orthogonal transformation of $R^{n+1}$, we may assume

$$
\left.f\right|_{S^{1}}(\cos \theta, \sin \theta, 0, \ldots, 0)=\left(f_{0}, f_{1}, \ldots, f_{n}\right)
$$

with derivatives $f_{0}^{\prime}=a, f_{1}^{\prime}=f_{2}^{\prime}=\cdots=f_{n}^{\prime}=0$, at $\theta=0$. Thus the function $f_{0}=f_{0}(\theta)$ is represented by trigonometric polynomial of degree at most $k$,

$$
f_{0}(\theta)=a_{0}+a_{1} \cos \theta+b_{1} \sin \theta+\cdots+a_{k} \cos k \theta+b_{k} \sin k \theta\left(a_{i}, b_{j} \in R\right) .
$$

Since $f_{0}^{2}+\cdots+f_{n}^{2}=1,\left|f_{0}(\theta)\right| \leq 1$, by the Bernstein-Szego Inequality, we finally obtain the vital inequality

$$
|a|=\left|f_{0}^{\prime}\right| \leq k \cdot \max \left|f_{0}\right| \leq k,
$$

and thus $\left|d f_{x}(V)\right|=|a| \leq k$.

Modelling on the proof of Theorem 1 in $[\mathbf{L a}]$, we may obtain:

Lemma 2.1. Let $f: S^{m} \rightarrow S^{n}$ be a 2-form (quadratic map). Assume moreover that $f$ is onto. Then $\delta(f) \geq 2$. 
Proof. Suppose $\delta(f)<2$. Take any point $x$ in $S^{m}$ so that $f(-x)=f(x)$. Then each meridian in $S^{m}$ from $x$ to $(-x)$ is mapped into a loop passing through $f(x)$ of length less than $2 \pi$. Hence $-f(x)$ does not lie in the image of $f$, and therefore $f$ is not onto.

Combining the preceding lemma with Theorem 1.1 we obtain at once:

Corollary 2.2. Let $f: S^{m} \rightarrow S^{n}$ be a 2-form. If $f$ is onto, then $\delta(f)=2$.

Remark 2.3. In [Ro], Roitberg proved his Theorem 2 by computing the dilatation of map $\bar{f}: S^{n+k} \rightarrow S^{n}$, defined by

$$
\bar{f}(a, b)=\left(2 g(a) \cdot b,|b|^{2}-|a|^{2}\right)
$$

for $a=\left(a_{1}, \ldots, a_{k+1}\right) \in R^{k+1}, b=\left(b_{1}, \ldots, b_{n}\right) \in R^{n}$, where $g\left(S^{k}\right) \subset O(n)$. Note that this map $\bar{f}$ is a quadratic map, the assertion $\delta(\bar{f})=2$ follows immediately from Corollary 2.2.

Theorem 1.1 provides upper bounds for the dilatation of polynomial maps between spheres. It is natural to ask the question: Given $m, n, k \in Z^{+}$, when does there exist a polynomial map $f: S^{m} \rightarrow S^{n}$ of degree $k$, with $\delta(f)=k$ ? If $m>n$, according to [Wo], it is in general not easy to solve this question. However, if $m=n$, we can answer this question affirmatively with the following:

Theorem 2.4. Let $f_{k}: S^{n} \rightarrow S^{n}(k>0)$ be defined by

$$
(\cos \theta, \sin \theta \cdot x) \mapsto(\cos k \theta, \sin k \theta \cdot x),
$$

where $0 \leq \theta \leq \pi$ and $x \in S^{n-1} \subset R^{n}$. Then $f_{k}$ can be characterized as follows:

(1) $f_{k}$ is actually a $k$-form;

(2) $\delta\left(f_{k}\right)=k$;

(3) the Brouwer degree of $f_{k}$ is given by

$$
\operatorname{deg} f_{k}= \begin{cases}k & \text { if } n \text { is odd, } \\ 1 & \text { if } n \text { is even and } k \text { is odd } \\ 0 & \text { otherwise. }\end{cases}
$$


Proof. (1) It suffices to observe that

$$
\begin{aligned}
\cos k \theta+i \sin k \theta= & (\cos \theta+i \sin \theta)^{k} \\
= & \sum_{j}\left(\begin{array}{c}
k \\
j
\end{array}\right) \cos ^{k-j} \theta \cdot \sin ^{j} \theta \cdot i^{j} \\
= & \sum_{p}(-1)^{p}\left(\begin{array}{c}
k \\
2 p
\end{array}\right) \cos ^{k-2 p} \theta \cdot|\sin \theta \cdot x|^{2 p} \\
& +\sum_{p}(-1)^{p} i\left(\begin{array}{c}
k \\
2 p+1
\end{array}\right) \cos ^{k-2 p-1} \theta \cdot|\sin \theta \cdot x|^{2 p} \cdot \sin \theta .
\end{aligned}
$$

(2) Since $f_{k}$ is a $k$-form, it follows from Theorem 1.1 that $\delta\left(f_{k}\right) \leq k$. It remains only to find a suitable point $x$ in $S^{n}$ and a suitable unit tangent vector $V$ to $x$ satisfying $\left|d\left(f_{k}\right)_{x}(V)\right|=k$. The work is not difficult and left to the reader.

(3) The conclusion can be proved in several way. We will make use of moving frames to calculate the Brouwer degree of $f_{k}$. Let $S^{n-1}=\left\{x \in R^{n} \mid\right.$ $\langle x, x\rangle=1\}$. We choose a local field of orthonormal frame $e_{1}, e_{2}, \ldots, e_{n-1}$ in $T S^{n-1}$. Define

$$
\omega_{i}=\left\langle d x, e_{i}\right\rangle, i=1,2, \ldots, n-1 .
$$

Then the volume element of $S^{n-1}$ can be written as

$$
d S^{n-1}=\omega_{1} \wedge \cdots \wedge \omega_{n-1} .
$$

Now write $S^{n}$ as

$$
S^{n}=\left\{y \in R^{n+1} \mid y=(\cos \theta, \sin \theta \cdot x), 0 \leq \theta \leq \pi, x \in S^{n-1}\right\} .
$$

Then

$$
\begin{aligned}
d y & =(d \cos \theta, d(\sin \theta \cdot x)) \\
& =(-\sin \theta, \cos \theta \cdot x) d \theta+(0, \sin \theta d x) \\
& =(-\sin \theta, \cos \theta \cdot x) d \theta+\sum_{i=1}^{n-1}\left(0, e_{i}\right) \sin \theta \cdot \omega_{i} .
\end{aligned}
$$

For convenience we write

$$
e_{0}=(-\sin \theta, \cos \theta \cdot x), e_{i}=\left(0, e_{i}\right), i=1,2, \ldots, n-1 .
$$

It is clear to see that $e_{0}, e_{1}, \ldots, e_{n-1}$ provides a local orthonormal frame field of $T S^{n}$. In addition, define

$$
\phi_{j}=\left\langle d y, e_{j}\right\rangle, j=0,1,2, \ldots, n-1 .
$$

We are led to

$$
\phi_{0}=d \theta, \phi_{j}=\sin \theta \cdot \omega_{i}(i=1,2, \ldots, n-1) .
$$


Further, we find

$$
\begin{aligned}
d \widetilde{y} & =d f_{k}(y) \\
& =d(\cos k \theta, \sin k \theta \cdot x) \\
& =(-\sin k \theta, \cos k \theta) k d \theta+\left(0, e_{i}\right) \sin k \theta \cdot \omega_{i} .
\end{aligned}
$$

It follows from the definition $d \widetilde{y}=\sum \psi_{j} \varepsilon_{j}$ that

$$
\psi_{0}=k d \theta, \psi_{i}=\sin k \theta \cdot \omega_{i}(i=1,2, \ldots, n-1) .
$$

By the definition of the Brouwer degree, we conclude that

$$
\begin{aligned}
\operatorname{deg} f_{k} \cdot V\left(S^{n}\right) & =\int_{S^{n}} f_{k}^{*} d \widetilde{V} \\
& =\int_{S^{n}} \psi_{0} \wedge \cdots \wedge \psi_{n-1} \\
& =\int_{S^{n}} k \sin ^{n-1} k \theta d \theta \wedge \omega_{1} \wedge \cdots \wedge \omega_{n-1},
\end{aligned}
$$

and hence

$$
\operatorname{deg} f_{k}=\frac{k \int_{0}^{\pi} \sin ^{n-1} k \theta d \theta}{\int_{0}^{\pi} \sin ^{n-1} \theta d \theta}
$$

from which the required result follows immediately.

We turn to investigate the exact value of the dilatation of gradient maps of isoparametric polynomials. Let $f$ be a homogeneous polynomial of degree $g$ on the Euclidean space $R^{n+2}$. Recall that $f$ is called an isoparametric polynomial if it satisfies the following Cartan-Münzner's differential equations:

$$
\begin{aligned}
|\nabla f|^{2} & =g^{2}|x|^{2 g-2}, \\
\triangle f & =\frac{1}{2} g^{2}\left(m_{2}-m_{1}\right)|x|^{g-2},
\end{aligned}
$$

where $\nabla f$ and $\triangle f$ denote the gradient and the Laplacian of $f$ respectively, and $m_{1}, m_{2}$ two (possible equal) natural numbers (see, for example, [PT1], [CR]). E. Cartan has solved completely the classification problem for isoparametric polynomials for $g=1,2$ or 3 . By using cohomological arguments, Münzner obtained the splendid result that the number $g$ of distinct principal curvatures of level sets can be only $1,2,3,4$ or 6 .

Note that the gradient map $\Phi$, defined by $\Phi=\frac{1}{k} \nabla f$, is a map from $R^{n+2}$ to $R^{n+2}$. Moreover, every component of $\Phi$ is a homogeneous polynomial of degree $g-1$. Since $f$ satisfies the Cartan-Münzner's differential equations, the restriction of $\Phi$ on the unit sphere produces a $(g-1)$-form from $S^{n+1}$ to $S^{n+1}$. In [PT1], the Brouwer degree of $\Phi$ was calculated. An analysis of 
the gradient map of isoparametric polynomial, given in [PT1], will imply the following:

Proposition 2.5. Let $\Phi=\frac{1}{k} \nabla f$ and $f$ be an isoparametric polynomial of degree $g$. Then $\delta(\Phi)=g-1$.

Proof. The conclusion follows obviously from (1.8) and (1.18) in [PT1].

To conclude this section we wish to pose a natural question: Given $n, k \in$ $Z^{+}$, how many equivalence classes (under orthorgonal transformations) of polynomial maps $f: S^{n} \rightarrow S^{n}$ of degree $k$ with $\delta(f)=k$ are there?

It is well-known that classifying isoparametric polynomials amounts classifying their gradient maps. Let $f_{1}$ and $f_{2}$ be the isoparametric polynomials of degree 4 with multiplicities $\left(m_{1}, m_{2}\right)=(1,3)$ and $(2,2)$, respectively. Obviously $f_{1}$ is not equivalent to $f_{2}$, hence the gradient maps $\frac{1}{4} \nabla f_{1}: S^{9} \rightarrow S^{9}$ and $\frac{1}{4} \nabla f_{2}: S^{9} \rightarrow S^{9}$ are not equivalent, although they have equal dilatation 3 .

For spheres of lower dimensions, we are able to show the following uniqueness result:

Proposition 2.6. Let $f: S^{2} \rightarrow S^{2}$ be a 3-form. If $\delta(f)=3$, then by suitable orthogonal transformations, $f$ coincides with the "standard" $f_{3}$ which is given in Theorem 2.4.

The proof is entirely elementary, but full of precise analysis. We wish to write down the detailed proof in a future paper.

\section{Differentiable maps.}

This section is devoted to the proofs of Propositions 1.2 and 1.3 and Theorem 1.4. We begin by stating the following approximation result.

Lemma 3.1. Suppose $a<c<b$, and $0 \leq k_{1}<k_{2}$. Let $f$ be a piecewise linear function defined by

$$
f(x)= \begin{cases}k_{1} x+d, & \text { if } x \in(a, c] ; \\ k_{2}(x-c)+\left(k_{1} c+d\right), & \text { if } x \in[c, b) .\end{cases}
$$

Then for any $\epsilon>0$ there exist $\delta>0$ and a differentiable function $\tilde{f} \epsilon$ $C^{1}(a, b)$ such that:

(1) $\tilde{f}(x)=f(x)$ for $x \in(a, c-\delta] \bigcup[c+\delta, b)$;

(2) $|\widetilde{f}(x)-f(x)|<\epsilon$ and $k_{1} \leq \tilde{f}^{\prime}(x) \leq k_{2}$ for $x \in(c-\delta, c+\delta)$.

Proof. Obvious.

We note that this lemma can be generalized to the general case where $f$ is a piecewise linear function of multiple pieces. 
Proof of Proposition 1.2. To prove $\delta(2,4) \leq 2 \sqrt{2}$, it suffices to construct a $C^{1}$-map $f: S^{2} \rightarrow S^{2}$ of the Brouwer degree 4 , with $\delta(f) \leq 2 \sqrt{2}+\epsilon$ for any given small $\epsilon>0$. The proof is divided into three steps.

(i) Write the two dimensional sphere $S^{2}$ as

$$
S^{2}=\left\{\left(\cos \theta, \sin \theta \cdot e^{i \phi}\right) \mid 0 \leq \theta \leq \pi, 0 \leq \phi \leq 2 \pi\right\} .
$$

$S^{2}$ is then divided into two parts

$$
S_{1}=\left\{0 \leq \theta \leq \frac{\pi}{2}\right\} \text { and } S_{2}=\left\{\frac{\pi}{2} \leq \theta \leq \pi\right\}
$$

whose intersection is precisely the equator.

In this way we define $F_{1}: S_{1} \rightarrow S^{2}$ by

$$
F_{1}\left(\cos \theta, \sin \theta \cdot e^{i \phi}\right)=\left(\cos 2 \theta, \sin 2 \theta \cdot e^{i 2 \phi}\right),
$$

and $F_{2}: S_{2} \rightarrow S^{2}$ by the composition: $F_{2}=F_{1} \circ j$, where $j: S^{2} \rightarrow S^{2}$ is defined by sending $\left(x_{1}, x_{2}, x_{3}\right) \in S^{2} \subset R^{3}$ to $\left(-x_{1}, x_{2},-x_{3}\right)$. We finally get a map $F: S^{2} \rightarrow S^{2}$ simply defined by $\left.F\right|_{S_{1}}=F_{1}$ and $\left.F\right|_{S_{2}}=F_{2}$. Since the diffeomorphism $j$ is orientation preserving, $F$ has the Brouwer degree 4. Intuitively, the map $F$ wraps the source $S^{2}$ around the target $S^{2}$ four times. We remark that $F$ is not differentiable.

(ii) Define $\widetilde{F}_{1}: S_{1} \rightarrow S^{2}$ by

$$
\widetilde{F}_{1}\left(\cos \theta, \sin \theta \cdot e^{i \phi}\right)=\left(\cos \alpha(\theta), \sin \alpha(\theta) \cdot e^{i 2 \phi}\right),
$$

where $\alpha:\left[0, \frac{\pi}{2}\right] \rightarrow[0, \pi]$ is a function given by

$$
\alpha(\theta)= \begin{cases}0, & \theta \in[0, \delta \pi] ; \\ \frac{5}{4-16 \delta} \cdot(x-\delta \pi), & \theta \in\left[\delta \pi, \frac{\pi}{4}\right] ; \\ \frac{11}{4-16 \delta} \cdot\left(x-\frac{\pi}{2}+\delta \pi\right)+\pi, & \theta \in\left[\frac{\pi}{4}, \frac{\pi}{2}-\delta \pi\right] ; \\ \pi, & \theta \in\left[\frac{\pi}{2}-\delta \pi, \frac{\pi}{2}\right] .\end{cases}
$$

Here $\delta>0$ is sufficiently small. In fact, $\alpha$ is a piecewise linear function joining the points $(0,0),(\delta \pi, 0),\left(\frac{\pi}{4}, \frac{5 \pi}{16}\right),\left(\frac{\pi}{2}-\delta \pi, \pi\right),\left(\frac{\pi}{2}, \pi\right)$ together.

Again, define $\widetilde{F}_{2}: S_{2} \rightarrow S^{2}$ by $\widetilde{F}_{2}=\widetilde{F}_{1} \circ j$. Furthermore, we have $\widetilde{F}: S^{2} \rightarrow S^{2}$ by simply defining

$$
\left.\widetilde{F}\right|_{S_{1}}=\widetilde{F}_{1} \text { and }\left.\widetilde{F}\right|_{S_{2}}=\widetilde{F}_{2} .
$$

It is easily seen that $\widetilde{F}$ is homotopic to $F$ whose Brouwer degree is equal to 4 . Hence $\operatorname{deg} \widetilde{F}=4$. On the other hand, it is evident to verify

$$
\sup _{\substack{0 \leq \theta \leq \frac{\pi}{2}, \theta \neq 0, \delta \pi, \frac{\pi}{2}-\delta \pi, \frac{\pi}{2}}}\left\{2\left|\frac{\sin \alpha(\theta)}{\sin \theta}\right|,\left|\alpha^{\prime}(\theta)\right|\right\} \leq 2 \sqrt{2}
$$

which implies that the dilatation of $\widetilde{F}$ on the domain of smooth points is not bigger than $2 \sqrt{2}$. It should be remarked that the particular $\alpha$ is not the 
optimal choice. In fact, one can improve the estimate $2 \sqrt{2}$ by altering $\alpha$. However, we don't know the minimum.

(iii) It remains to uniformly approximate $\widetilde{F}$ by a $C^{1}$ map $f$ such that $\delta(f) \leq 2 \sqrt{2}+\epsilon$. Of course, approximating does not change the homotopy class, and then preserves the Brouwer degree. The desired map is guaranteed by applying a variation of Lemma 3.1 .

The proof of Proposition 1.2 is now complete.

Remark 3.2. By making use of the sphere-packing method of Gromov in [Gr2], one may get that $\delta(2,4) \leq \frac{\pi}{\arcsin \sqrt{\frac{2}{3}}} \approx 3.289$. More precisely, inside the unit sphere $S^{2}$, there exists an inscribed regular tetrahedron with four vertices: $(0,0,1),\left(\frac{\sqrt{8}}{3}, 0,-\frac{1}{3}\right),\left(-\frac{\sqrt{2}}{3}, \sqrt{\frac{2}{3}},-\frac{1}{3}\right)$ and $\left(-\frac{\sqrt{2}}{3},-\sqrt{\frac{2}{3}},-\frac{1}{3}\right)$. Taking these four vertices as centers, we can construct four geodesic spheres tangent to each other. It is straitforward to show $\rho=\arcsin \sqrt{\frac{2}{3}}$. Wrapping the four geodesic spheres around the target $S^{2}$ by geodesic streching respectively (see [Gr2] for details), we obtain a map $f: S^{2} \rightarrow S^{2}$ such that $\operatorname{deg} f=4$ and $\delta(f)=\frac{\pi}{\rho}$. By contrast with this conclusion, Hefter $[\mathbf{H e}]$ stated that $\delta(2,4)<3$ without detailed proof. On the other side, since $S^{2}$ is simply connected, and thus any map $f$ of degree different from \pm 1 has at least one critical point, it follows from an integration inequality $[\mathbf{G r 2}$, p. 14] that there is no $C^{1}$ map $f: S^{2} \rightarrow S^{2}$ satisfying both $\operatorname{deg} f=4$ and $\delta(f)=2$.

To estimate the value of $\delta(3,4)$, we first point out a mistake in [Ol, p. 389] which claimed that $\delta(F)=2$, where $F$ is given as follows:

Proposition 3.3. As in $[\mathrm{Ol}]$, define $F: S^{3} \rightarrow S^{3}$ by

$$
F(Z, W)=\left(\frac{Z^{2}}{\sqrt{|Z|^{4}+|W|^{4}}}, \frac{W^{2}}{\sqrt{|Z|^{4}+|W|^{4}}}\right)
$$

for $(Z, W) \in S^{3} \subset C \times C$. Then $\delta(F) \geq \sqrt{\frac{24}{5}}>2$.

Proof. Take $x=\left(\frac{\sqrt{3}}{2}, 0, \frac{1}{2}, 0\right)$ in $S^{3}$ and $V=(0,1,0,0)$ a tangent vector to $x$. It is not difficult to verify

$$
\left|d(F)_{x}(V)\right|^{2}=\frac{24}{5}
$$

as required.

This result is not special. In fact, we can prove that $\delta\left(f_{\alpha}\right)>2$ for general differentiable join $f_{\alpha}$. More precisely, we state: 
Proposition 3.4. Define $f_{\alpha}: S^{3} \rightarrow S^{3}$ by

$$
f_{\alpha}\left(\cos \theta \cdot e^{i \phi}, \sin \theta \cdot e^{i \psi}\right)=\left(\cos \alpha(\theta) \cdot e^{i 2 \phi}, \sin \alpha(\theta) \cdot e^{i 2 \psi}\right)
$$

for $\left(\cos \theta \cdot e^{i \phi}, \sin \theta \cdot e^{i \psi}\right) \in S^{3} \subset C \times C$, where $\alpha=\alpha(\theta):\left[0, \frac{\pi}{2}\right] \rightarrow\left[0, \frac{\pi}{2}\right]$ is a function so that $f_{\alpha}$ is differentiable. Then $\operatorname{deg} f_{\alpha}=4$ and $\delta\left(f_{\alpha}\right)>2$.

Proof. As mentioned earlier, $f_{\alpha}$ is homotopic to $f: S^{3} \rightarrow S^{3}$ defined by

$$
f\left(\cos \theta \cdot e^{i \phi}, \sin \theta \cdot e^{i \psi}\right)=\left(\cos \theta \cdot e^{i 2 \phi}, \sin \theta \cdot e^{i 2 \psi}\right)
$$

whose Brouwer degree is easily seen to equal to 4 . Therefore we obtain $\operatorname{deg} f_{\alpha}=4$. Suppose now that $\delta\left(f_{\alpha}\right) \leq 2$. It follows that $\left|d f_{\alpha}\left(\frac{\partial}{\partial \phi}\right)\right| \leq$ $2\left|\frac{\partial}{\partial \phi}\right|$. Since $\frac{\partial}{\partial \phi}=\left(\cos \theta \cdot i e^{i \phi}, 0\right)$ and $d f_{\alpha}\left(\frac{\partial}{\partial \phi}\right)=\left(2 \cos \alpha(\theta) \cdot i e^{i 2 \phi}, 0\right)$, we have therefore that $|\cos \alpha(\theta)| \leq|\cos \theta|$ for $\theta \in\left[0, \frac{\pi}{2}\right]$, equivalently $\alpha(\theta) \equiv$ $\theta$. Hence $f_{\alpha}$ coincides $f$, which is not differentiable. This contradicts the assumption.

Remark 3.5. The function $\alpha(\theta)$ in [Ol, p. 389] is given by

$$
\alpha(\theta)=\arccos \frac{\cos ^{2} \theta}{\sqrt{\cos ^{4} \theta+\sin ^{4} \theta}} .
$$

It seems that one does not know if there is a $C^{1}$ map $f: S^{3} \rightarrow S^{3}$ of the Brouwer degree 4 and $\delta(f)=2$. However, we have the following:

Lemma 3.6. For any small $\epsilon>0$, there exists a $C^{1}$ map $f_{\alpha}: S^{3} \rightarrow S^{3}$ such that $\operatorname{deg} f_{\alpha}=4$ and $\delta\left(f_{\alpha}\right) \leq 2+\epsilon$.

Proof. Define $f_{\alpha}: S^{3} \rightarrow S^{3}$ by

$$
f_{\alpha}\left(\cos \theta \cdot e^{i \phi}, \sin \theta \cdot e^{i \psi}\right)=\left(\cos \alpha(\theta) \cdot e^{i 2 \phi}, \sin \alpha(\theta) \cdot e^{i 2 \psi}\right),
$$

where $\alpha=\alpha(\theta):\left[0, \frac{\pi}{2}\right] \rightarrow\left[0, \frac{\pi}{2}\right]$ satisfies $\alpha(0)=0$ and $\alpha\left(\frac{\pi}{2}\right)=\frac{\pi}{2}$. As mentioned before, the Brouwer degree of $f_{\alpha}$ is exactly 4. Assume that $f_{\alpha}$ is differentiable. Since $\frac{\partial}{\partial \phi}=\left(\cos \theta \cdot i e^{i \phi}, 0\right), \frac{\partial}{\partial \psi}=\left(0, \sin \theta \cdot i e^{i \psi}\right), \frac{\partial}{\partial \theta}=$ $\left(-\sin \theta \cdot e^{i \phi}, \cos \theta \cdot e^{i \psi}\right)$, and then

$$
\delta\left(f_{\alpha}\right)=\sup _{0 \leq \theta \leq \frac{\pi}{2}}\left\{2\left|\frac{\cos \alpha(\theta)}{\cos \theta}\right|, 2\left|\frac{\sin \alpha(\theta)}{\sin \theta}\right|,\left|\alpha^{\prime}(\theta)\right|\right\} .
$$

The inequality $\delta\left(f_{\alpha}\right) \leq 2+\epsilon$ is therefore equivalent to the following inequalities:

$$
\begin{aligned}
\left|\frac{\cos \alpha(\theta)}{\cos \theta}\right| & \leq 1+\frac{\epsilon}{2} \\
\left|\frac{\sin \alpha(\theta)}{\sin \theta}\right| & \leq 1+\frac{\epsilon}{2} \\
\left|\alpha^{\prime}(\theta)\right| & \leq 2+\epsilon .
\end{aligned}
$$


Finally, the existence of $\lim _{\theta \rightarrow 0} \frac{\sin \alpha(\theta)}{\sin ^{2}(\theta)}$ and $\lim _{\theta \rightarrow \frac{\pi}{2}} \frac{\cos \alpha(\theta)}{\cos ^{2}(\theta)}$ guarantee that $f$ is a $C^{1}$ map. We may construct such a satisfactory function $\alpha=\alpha(\theta)$ in two steps.

(1) For odd $n$, let $\beta=\beta(t)=\frac{1}{2 n} t^{n}\left(1-t^{2 n}\right), t \in[-1,1]$. Then, the following properties are easily verified for $t \in[-1,1]$ :

(1a) $\beta( \pm 1)=\beta(0)=0$;

(1b) $\beta^{\prime}( \pm 1)=-1,\left|\beta^{\prime}(t)\right| \leq 1$, and $\beta^{\prime}(t)=-1$ if and only if $t= \pm 1$;

(1c) $|\beta(t)| \leq \frac{1}{2 n}$;

(1d) $-1 \leq t+\beta(t) \leq 1$.

(2) Furthermore, let $\alpha=\alpha(\theta)=\theta+\frac{\pi}{4} \beta\left(\frac{4}{\pi} \theta-1\right)$ for $\theta \in\left[0, \frac{\pi}{2}\right]$. Then it follows from (1) that

(2a) $\alpha(0)=0, \alpha\left(\frac{\pi}{4}\right)=\frac{\pi}{4}, \alpha\left(\frac{\pi}{2}\right)=\frac{\pi}{2}$;

(2b) $\quad \alpha^{\prime}(0)=0, \alpha^{\prime}\left(\frac{\pi}{2}\right)=0$, and $\left|\alpha^{\prime}(\theta)\right| \leq 2$;

(2c) $\left|\frac{\pi}{4} \beta\left(\frac{4}{\pi} \theta-1\right)\right| \leq \frac{\pi}{8 n}$;

(2d) $0 \leq \alpha(\theta) \leq \theta$ if $\theta \in\left[0, \frac{\pi}{4}\right]$, and $\theta \leq \alpha(\theta) \leq \frac{\pi}{2}$ if $\theta \in\left[\frac{\pi}{4}, \frac{\pi}{2}\right]$.

Summarizing the above arguments, we have finally

$$
\begin{aligned}
&\left|\frac{\sin \alpha(\theta)}{\sin \theta}\right| \leq 1+\sin \frac{\pi}{8 n} \\
&\left|\frac{\cos \alpha(\theta)}{\cos \theta}\right| \leq 1+\sin \frac{\pi}{8 n} ; \\
&\left|\alpha^{\prime}(\theta)\right| \leq 2 ; \\
& \lim _{\theta \rightarrow 0} \frac{\sin \alpha(\theta)}{\sin ^{2} \theta} \text { and } \lim _{\theta \rightarrow \frac{\pi}{2}} \frac{\cos \alpha(\theta)}{\cos ^{2} \theta} \text { exist. }
\end{aligned}
$$

These imply that $(* 1),(* 2)$ and $(* 3)$ hold, if $n$ is sufficiently large. We get the desired function $\alpha=\alpha(\theta)$, and hence the proof of the lemma.

Proposition 1.3 is an immediate consequence.

We should mention that Gromov in [Gr2] gave a map $g: S^{3} \rightarrow S^{3}$ by $g\left(\cos \theta \cdot e^{i \phi}, \sin \theta \cdot e^{i \psi}\right)=\left(\cos \theta \cdot e^{i 2 \phi}, \sin \theta \cdot e^{i 2 \psi}\right)$ whose dilatation is equal to 2 and the Brouwer degree is just 4 . However, this map $g$ is only continuous, not differentiable. Note that for continuous map, the dilatation is defined by Lipschitz constant insead of $\sup \{|d f(V)||| V \mid=1\}$ (for more details, see $[\mathbf{H e}])$. Indeed, Lemma 3.6 provides explicitly a smooth approximation to the map $g$ of Gromov.

The last part of this section will be devoted to:

Proof of Theorem 1.4. Define $f: S^{3} \rightarrow S^{3}$ by

$$
f\left(\cos \theta \cdot e^{i \phi}, \sin \theta \cdot e^{i \psi}\right)=\left(\cos \theta \cdot e^{i 2 \phi}, \sin \theta \cdot e^{i \psi}\right)
$$


and denote by $\tau$ the homotopy class of $f$. It is easy to see that $\operatorname{deg} f=2$, and it follows from [O1, p. 389] that $\delta(\tau)=2$.

Next, define $\pi_{\alpha}: S^{3} \rightarrow S^{2}$ by

$$
\pi_{\alpha}\left(\cos \theta \cdot e^{i \phi}, \sin \theta \cdot e^{i \psi}\right)=\left(\cos \alpha(\theta), \sin \alpha(\theta) \cdot e^{i(\phi+\psi)}\right)
$$

where $\alpha:\left[0, \frac{\pi}{2}\right] \rightarrow[0, \pi]$ satifies $\alpha(0)=0, \alpha\left(\frac{\pi}{2}\right)=\pi$. Note that if $\alpha(\theta) \equiv 2 \theta$, then $f_{\alpha}$ is precisely the Hopf fibration $\pi: S^{3} \rightarrow S^{2}$. Moreover, any such map $\pi_{\alpha}$ (called $\alpha$-Hopf construction) is homotopic to the Hopf fibration (see, for example, $[\mathbf{P T 2}]$ and $[\mathbf{B a}])$. It follows from Theorem 1 in [La, p. 433] that $\delta(\sigma)=2$ for $\sigma=\left[\pi_{\alpha}\right]=[\pi] \in \pi_{3} S^{2}$.

Now observe that the composition $\pi_{\alpha} \circ f: S^{3} \rightarrow S^{2}$ sends $\left(\cos \theta \cdot e^{i \phi}, \sin \theta\right.$. $\left.e^{i \psi}\right)$ in $S^{3}$ to $\left(\cos \alpha(\theta), \sin \alpha(\theta) \cdot e^{i(2 \phi+\psi)}\right)$ in $S^{2}$. Using a similar argument as before, we get

$$
\begin{aligned}
& \delta\left(\left.\pi_{\alpha} \circ f\right|_{\left.0<\theta<\frac{\pi}{2}\right)}\right. \\
& =\sup _{0<\theta<\frac{\pi}{2}}\left\{\left|\sqrt{a_{1}^{2}\left(\alpha^{\prime}(\theta)\right)^{2}+\left(a_{2} \frac{2 \sin \alpha(\theta)}{\cos \theta}+a_{3} \frac{\sin \alpha(\theta)}{\sin \theta}\right)^{2}}\right| a_{1}^{2}+a_{2}^{2}+a_{3}^{2}=1\right\} .
\end{aligned}
$$

We are then left to construct a suitable function $\alpha=\alpha(\theta)$ such that the composition $\pi \circ f$ is differentiable and the inequality $\delta\left(\pi_{\alpha} \circ f\right)<3$ holds.

Let $\delta>0$ be sufficiently small, and define $\alpha=\alpha(\theta)$ by

$$
\alpha(\theta)= \begin{cases}0, & \theta \in[0, \delta \pi] ; \\ (\theta-\delta \pi) \cdot \frac{3-16 \delta}{1-4 \delta}, & \theta \in\left[\delta \pi, \frac{\pi}{4}\right] ; \\ \left(\theta-\frac{\pi}{2}+\delta \pi\right) \cdot \frac{1+16 \delta}{1-4 \delta}+\pi, & \theta \in\left[\frac{\pi}{4}, \frac{\pi}{2}-\delta \pi\right] ; \\ \pi, & \theta \in\left[\frac{\pi}{2}-\delta \pi, \frac{\pi}{2}\right] .\end{cases}
$$

In other words, $\alpha$ is a piecewise linear function joining the points $(0,0)$, $(\delta \pi, 0),\left(\frac{\pi}{4}, \frac{3}{4} \pi-4 \delta \pi\right),\left(\frac{\pi}{2}-\delta \pi, \pi\right),\left(\frac{\pi}{2}, \pi\right)$ together. It is evident to see that the function $\alpha$ has slopes $0, \frac{3-16 \delta}{1-4 \delta}(<3), \frac{1+16 \delta}{1-4 \delta}\left(<\frac{3}{2}\right)$ and 0 on the four intervals, respectively. It is straitforward to verify that

$$
\begin{gathered}
\sin ^{2} \alpha(\theta) \leq \frac{3}{2} \sin 2 \theta \\
\sup _{\theta \in\left(0, \frac{\pi}{2}\right), \theta \neq \delta \pi, \frac{\pi}{4}, \frac{\pi}{2}-\delta \pi}\left\{\left|\alpha^{\prime}(\theta)\right|, 2\left|\frac{\sin \alpha(\theta)}{\cos \theta}\right|,\left|\frac{\sin \alpha(\theta)}{\sin \theta}\right|\right\}<3 .
\end{gathered}
$$

Hence $\delta\left(\pi_{\alpha} \circ f \mid 0<\theta<\pi / 2\right) \leq \sqrt{15}$. It remains to make a smooth approximation to the map $\pi_{\alpha} \circ f$. Applying a variation of Lemma 3.1 will produce the desired map, which lies in the homotopy class $\sigma \circ \tau \in \pi_{3} S^{2}$ with dilatation less than 3 . The proof is now complete.

To conclude this section we wish to point out that Theorem 1.4 still holds for $\sigma \in \pi_{7} S^{4}$ (or $\pi_{15} S^{8}$ ) the Hopf class and $\tau \in \pi_{7} S^{7}$ (or $\pi_{15} S^{15}$ ) of the Brouwer degree 2. The proof is entirely analogous to that for $\pi_{3} S^{2}$. 
Acknowledgements. The authors are very grateful to Professor S.S. Chern and Professor M. Gromov for their encouragements, to Ms. Guan for careful typing, and to referee for nice comments. The second named author thanks Professor Yu J.T. for inviting to visit Hong Kong University. The research was supported by The Hong Kong Qiu-Shi Foundation, The National Natural Science Foundation of China and Academia Sinica, as well as the Education Foundation of Tsinghua University.

\section{References}

[Ba] P. Baird, Harmonic maps with symmetry, harmonic morphisms and deformations of metrics, Res. Notes in Math., 87, Pitman, London, 1983, MR 85i:58038, Zbl 0515.58010.

[BE] P. Borwein and T. Erdélyi, Polynomials and polynomial inequalities, GTM, 161, Springer-Verlag, 1995, MR 97e:41001, Zbl 0840.26002.

[CR] T.E. Cecil and P.J. Ryan, Tight and taut immersions of manifolds, Res. Notes in Math., 107, Pitman, Boston, 1985, MR 87b:53089, Zbl 0596.53002.

[Gr1] M. Gromov, Homotopical effects of dilatation, J. Differ. Geom., 13 (1978), 303-310, MR 82d:58017, Zbl 0427.58010.

[Gr2] Structures Métriques pour les Variétés Riemanniennes, CEDIC, Paris, 1981, MR 85e:53051, Zbl 0509.53034.

[He] H. Hefter, Dehnungsuntersuchungen an sphärenabbildungen, Invent. Math., 66 (1982), 1-10, MR 84a:55012, Zbl 0526.55015.

[La] H.B. Lawson, Stretching phenomena in mappings of spheres, Proc. Amer. Math. Soc., 19 (1968), 433-435, MR 37 \#5886, Zbl 0161.42702.

[Ol] R. Olivier, Über die dehnung von sphärenabbildungen, Invent. Math., 1 (1966), 380-390, MR 34 \#3500, Zbl 0146.19802.

[PT1] C.K. Peng and Z.Z. Tang, The Brouwer degrees of the gradient maps of isoparametric functions, Science in China, 39 (1996), 1131-1139, MR 98c:58039, Zbl 0874.58013.

[PT2] _ On representing homotopy classes of spheres by harmonic maps, Topology, 36 (1997), 867-879, MR 98a:58053, Zbl 0873.58024.

[PT3] _ Harmonic maps from spheres to spheres, Topology, 37 (1998), 39-44, MR 99b:58064, Zbl 0889.55007.

[PT4] _ Algebraic maps between spheres, Science in China, 42 (1999), 1147-1154, MR 2000m:55015.

[Ro] J. Roitberg, The dilatation and filtration invariant in the homotopy of spheres, Adv. in Math., 20 (1976), 280-284, MR 54 \#6138, Zbl 0324.55017.

[Wo] R. Wood, Polynomial maps from spheres to spheres, Invent. Math., 5 (1968), 163168, MR 37 \#3583, Zbl 0204.23805.

Received August 11, 2000 and revised February 18, 2001.

Department of Mathematics, Graduate School

Academia Sinica

Beijing 100039, China 
E-mail address: pengck@sun.ihep.ac.cn

Department of Mathematical Sciences

Tsinghua University

BeiJing 100084, China

E-mail address: zztang@mx.cei.gov.cn 